\title{
Implementation of Prognostic Methodologies to Cryogenic Propellant Loading Testbed
}

\author{
Chetan S. Kulkarni*, Matthew Daigle ${ }^{\dagger}$, and Kai Goebel ${ }^{\dagger}$ \\ *SGT Inc., NASA Ames Research Center, Moffett Field,CA, 94035, USA \\ chetan.s.kulkarni@nasa.gov \\ ${ }^{\dagger}$ NASA Ames Research Center, Moffett Field,CA, 94035, USA \\ matthew.j.daigle@nasa.gov \\ kai.goebel@nasa.gov
}

\begin{abstract}
Prognostics methodologies determine the health state of a system and predict the end of life and remaining useful life. This information enables operators to take maintenancerelated decisions, thus effectively streamlining operational and mission-level activities. Prognostics testbeds help in the prototyping, development, and maturation of prognostic technologies. In this work, we present a prognostics testbed for pneumatic valves. Pneumatic valves are critical components in many industrial processes, and the testbed will be used to showcase how remaining life prediction works in the context of cryogenic refueling operations. The testbed allows for the injection of time-varying leaks with specified damage progression profiles in order to emulate common valve faults. In addition, the testbed contains a battery used to power some components, allowing the study of the effects of battery degradation on the operation of the valves. Prognostic algorithms will utilize sensor data collected from the different transducers in order to estimate component health and make life predictions, based on mathematical models describing the underlying physics of component degradation and employing a Bayesian filtering algorithm for state-parameter estimation from which life predictions are made.
\end{abstract}

\section{INTRODUCTION}

Prognostics methodologies determine the health state of a component or subsystem, and predict the end of life (EOL) and remaining useful life (RUL). This information enables operators to take maintenance-related decisions, thus effectively streamlining operational and mission-level activities. In cryogenic propellant loading operations, ensuring system safety through prognostics and health management technologies is critical to maintaining launch availability and reducing maintenance cost [1]. In order to mature propellant loading technologies, a testbed has been developed at NASA Kennedy Space Center (KSC) that transfers cryogenic propellant from a storage tank to a vehicle tank through a network of pipes, pumps, and valves [2].

Pneumatic-actuated valves play a critical role in such a system [3]; since the valves are used to control the flow of propellant, failures may have a significant impact on launch availability. In order to demonstrate prognostics in the context of cryogenic propellant loading, we have developed a prognostics demonstration testbed for pneumatic valves which allows the controlled injection of faults with varying degrees on a subset of valves of the larger testbed. In this paper, we describe the design and development of the testbed, which consists of two different types of pneumatic valves that allows the injection of faulty behavior and controllable fault progression. One valve type opens discretely, and is controlled through a solenoid valve; the other valve type opens continuously, and is controlled through a current-pressure transducer. Controllable leaks of pneumatic gas with specified damage progression profiles are introduced in the testbed through proportional valves, allowing the testing and validation of prognostics algorithms for pneumatic valves. The demonstration testbed will integrate with the cryogenic testbed at KSC through pneumatic ports and electrical connections.

The demonstration testbed also includes batteries that are used to power some components. Batteries are finding an increased use as backup power supply sources for critical systems and scientific applications all over the world. For these systems to operate at maximum efficiency and reliability, it becomes crucial to both monitor battery health and performance and to predict end of discharge (EOD) and EOL events. The testbed allows to study the effects of battery degradation on the operation of the control valves, as well as the prediction of EOD.

The structure of the paper is as follows. Section II presents the overall prognostics approach to the system using a modelbased methodology. Section III discusses the overall setup of the prognostics demonstration testbed. Section IV and V describe the leak and battery faults injected in the system, respectively. The paper ends with discussion and conclusions in Section VI.

\section{Prognostics Approach}

In this section, we first describe the general model-based prognostics problem, followed by an implementation architecture.

\section{A. Problem Formulation}

We assume the system model may be generally defined as

$$
\begin{aligned}
\mathbf{x}(k+1) & =\mathbf{f}(k, \mathbf{x}(k), \boldsymbol{\theta}(k), \mathbf{u}(k), \mathbf{v}(k)), \\
\mathbf{y}(k) & =\mathbf{h}(k, \mathbf{x}(k), \boldsymbol{\theta}(k), \mathbf{u}(k), \mathbf{n}(k)),
\end{aligned}
$$

where $k$ is the discrete time variable, $\mathbf{x}(k) \in \mathbb{R}^{n_{x}}$ is the state vector, $\boldsymbol{\theta}(k) \in \mathbb{R}^{n_{\theta}}$ is the unknown parameter vector, $\mathbf{u}(k) \in$ $\mathbb{R}^{n_{u}}$ is the input vector, $\mathbf{v}(k) \in \mathbb{R}^{n_{v}}$ is the process noise 
Prognostics

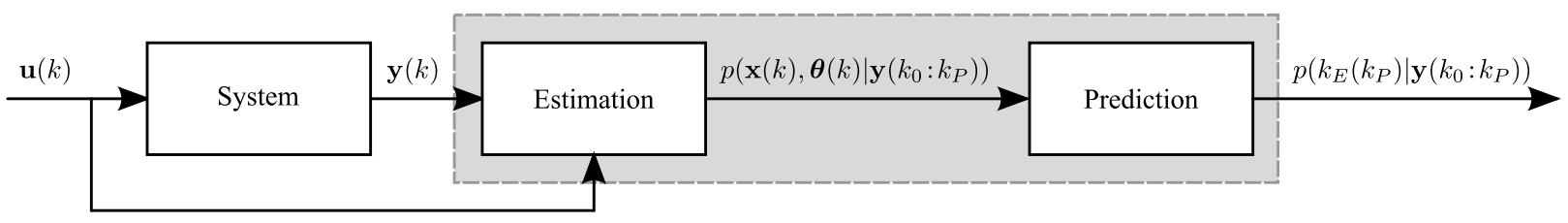

Fig. 1. Prognostics architecture.

vector, $\mathbf{f}$ is the state equation, $\mathbf{y}(k) \in \mathbb{R}^{n_{y}}$ is the output vector, $\mathbf{n}(k) \in \mathbb{R}^{n_{n}}$ is the measurement noise vector, and $\mathbf{h}$ is the output equation. ${ }^{1}$

In prognostics, we are interested in predicting the occurrence of some event $E$ that is defined with respect to the states, parameters, and inputs of the system. We define the event as the earliest instant that some event threshold $T_{E}: \mathbb{R}^{n_{x}} \times \mathbb{R}^{n_{\theta}} \times \mathbb{R}^{n_{u}} \rightarrow \mathbb{B}$, where $\mathbb{B} \triangleq\{0,1\}$ changes from the value 0 to 1 . That is, the time of the event $k_{E}$ at some time of prediction $k_{P}$ is defined as

$k_{E}\left(k_{P}\right) \triangleq \inf \left\{k \in \mathbb{N}: k \geq k_{P} \wedge T_{E}(\mathbf{x}(k), \boldsymbol{\theta}(k), \mathbf{u}(k))=1\right\}$.

The time remaining until that event, $\Delta k_{E}$, is defined as

$$
\Delta k_{E}\left(k_{P}\right) \triangleq k_{E}\left(k_{P}\right)-k_{P}
$$

In the context of systems health management, $T_{E}$ is defined via a set of performance constraints that define what the acceptable states of the system are, based on $\mathbf{x}(k), \boldsymbol{\theta}(k)$, and $\mathbf{u}(k)$ [4]. For valves, timing requirements are provided that define the maximum allowable time a valve may take to open or close [3]. For batteries, there are two types of predictions are relevant. The first is EOD, defined by a voltage threshold $V_{E O D}$. In this case, $T_{E}$ is defined by $V<V_{E O D}$, that is, when the battery voltage is less than the cutoff voltage, EOD is reached and $T_{E}$ evaluates to 1 . The second type of prediction is EOL, which is typically defined by a lower bound on the effective battery capacity [5]. In this case, $T_{E}$ is defined by $C<C_{E O L}$, where $C$ is the measured battery capacity and $C_{E O L}$ is the lower bound on capacity.

Models of the system components are constructed in this paradigm that capture both nominal behavior, as well as faulty behavior and damage progression[3]. Using these models, observations can be mapped back to the health state of the system as represented in $\mathbf{x}$ and $\boldsymbol{\theta}$. An estimation algorithm, such as the Kalman filter, unscented Kalman filter, or particle filter, is used to solve this problem [6]. This state-parameter estimate, along with a prediction of the future usage of the component, is used as input to a prediction algorithm that computes EOL and RUL [4], [7]. In order to account for fault effects propagating across different components, such as a leak through a solenoid valve causing changes in pneumatic valve behavior, a systemlevel perspective is needed for prognostics [8].

\footnotetext{
${ }^{1}$ Bold typeface denotes vectors, and $n_{a}$ denotes the length of a vector $\mathbf{a}$.
}

\section{B. Prognostics Architecture}

In a model-based prognostics architecture [4], there are two sequential problems, $(i)$ the estimation problem, which requires determining a joint state-parameter estimate $p\left(\mathbf{x}(k), \boldsymbol{\theta}(k) \mid \mathbf{y}\left(k_{0}: k\right)\right)$ based on the history of observations up to time $k, \mathbf{y}\left(k_{0}: k\right)$, and (ii) the prediction problem, which determines at $k_{P}$, using $p\left(\mathbf{x}(k), \boldsymbol{\theta}(k) \mid \mathbf{y}\left(k_{0}: k\right)\right)$, a probability distribution $p\left(k_{E}\left(k_{P}\right) \mid \mathbf{y}\left(k_{0}: k_{P}\right)\right)$. The distribution for $\Delta k_{E}$ can be trivially computed from $p\left(k_{E}\left(k_{P}\right) \mid \mathbf{y}\left(k_{0}: k_{P}\right)\right)$ by subtracting $k_{P}$.

The prognostics architecture is shown in Fig. 1 [4]. In discrete time $k$, the system is provided with inputs $\mathbf{u}_{k}$ and provides measured outputs $\mathbf{y}_{k}$. The estimation module uses this information, along with the system model, to compute an estimate $p\left(\mathbf{x}(k), \boldsymbol{\theta}(k) \mid \mathbf{y}\left(k_{0}: k\right)\right)$. The prediction module uses the joint state-parameter distribution and the system model, along with hypothesized future inputs, to compute the probability distribution $p\left(k_{E}\left(k_{P}\right) \mid \mathbf{y}\left(k_{0}: k_{P}\right)\right)$ at given prediction times $k_{P}$.

\section{OVERALl SETUP}

The prognostics demonstration testbed as shown in Figure 2 has been developed to demonstrate remaining life prediction of valves and batteries in the context of cryogenic refueling operations. The schematic shows the complete setup being developed for demonstration. The dashed lines are the electrical signals including the data acquisition I/O signals, power lines etc. The solid lines are all the pneumatic pressure lines connecting the control valves, solenoids, IPT etc. The schematic also illustrates the switching from normal supply to battery backup supply as well as a fail-safe mode to isolate the prognostics demonstration testbed from the cryogenic testbed.

As can be seen from the schematic diagram, two types of pneumatically-actuated valves are used for demonstration. The discrete-controlled valve (DV) illustrated in Figure 3 is a normally-closed valve with a linear cylinder actuator. The valve is opened by filling the chamber below the piston with gas up to the supply pressure, and evacuating the chamber above the piston down to atmospheric pressure. The valve is closed by filling the chamber above the piston, and evacuating the chamber below the piston. The return spring ensures that when pressure is lost, the valve will close due to the force exerted by the return spring, hence it is a normally-closed valve [3].

The continuous-controlled $(\mathrm{CV})$ valve as Figure 4 , on the other hand, opens in a continuous manner. Like the DV 


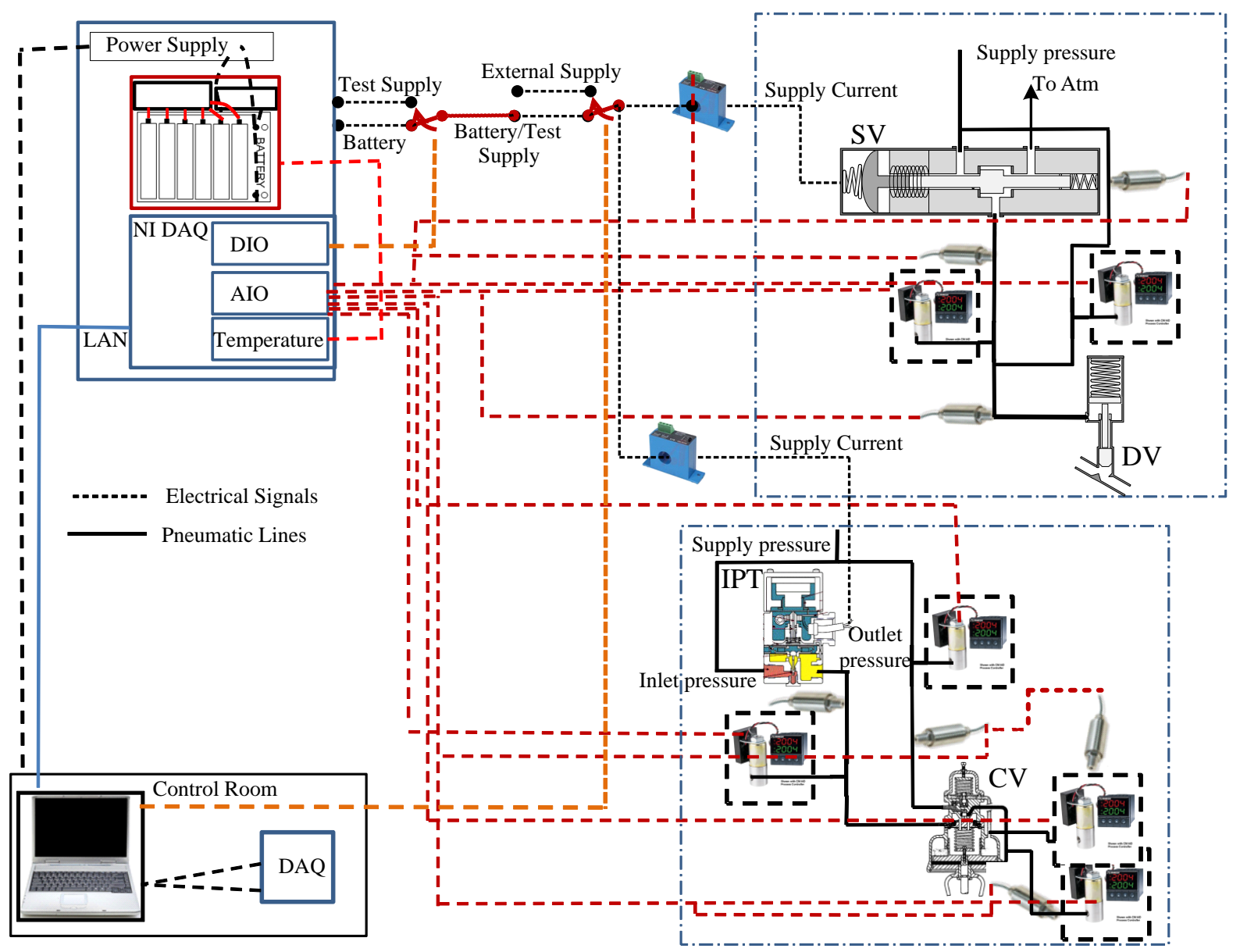

Fig. 2. Schematic for prognostics demonstration testbed setup

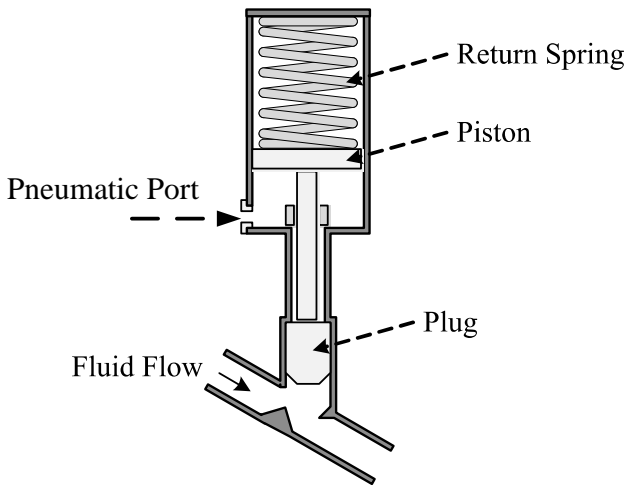

Fig. 3. Discrete-controlled valve

valve, the actuator contains a piston, however internally the actuator contains additional components in order to modulate the pressure applied to the piston. The actuator has two pressure ports, one for the supply pressure, and one for the signal pressure. The signal pressure is controlled between 3-15 psig in order to move the valve between fully closed and fully open. The actuator contains a 3-way, spool type pilot valve. Supply pressure is applied to one end of the spool while the other end of the spool is vented to the atmosphere through a diaphragm assembly. As the valve moves up or down as directed by the imbalance between the diaphragm and rate of spring forces, the valve spool either vents the positioner output port to the atmosphere or admits supply pressure to the positioner output port. Depending on the pilot valve position, the output pressure can vary from zero to full supply pressure, actuating the valve.

A three-way two-position solenoid valve (SV) is illustrated in Figure 5 is used for controlling the operation of the DV valve. The cylinder port connects to the valve, the normally closed (NC) port connects to the supply pressure, and normally open (NO) port is left unconnected, allowing venting to atmosphere. When the solenoid is energized, the path from the NC port to cylinder port is open, allowing gas to pass from the supply to the valve, thus actuating the valve. When deenergized, the supply pressure is closed off and the path from the cylinder port to the NO port is opened, thus venting the DV valve which closes the valve due to the return spring. The solenoid is powered by $24 \mathrm{~V} \mathrm{dc}$ either through the power supply or the Li-ion batteries for operation.

A current to pressure transducer (IPT) converts an analog current signal (4-20 mA) to a proportional linear pneumatic 


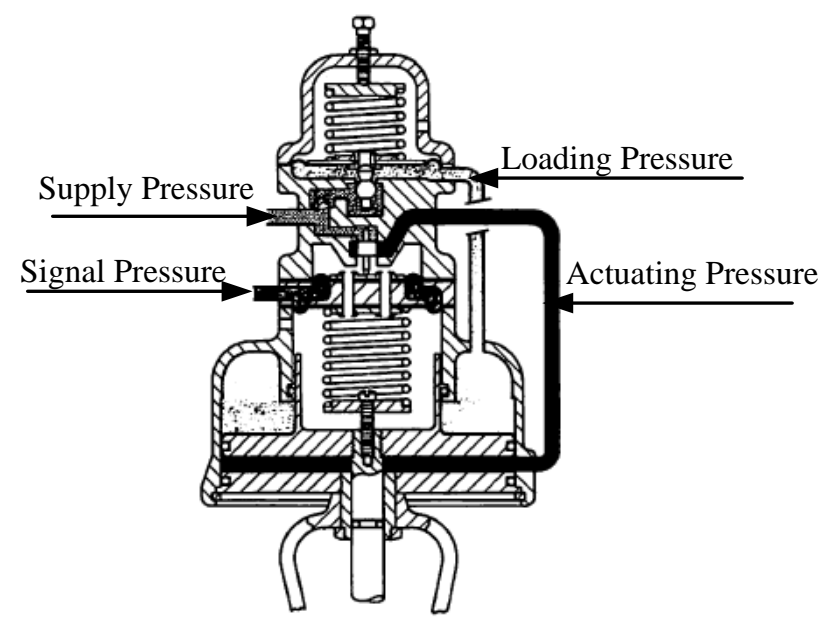

Fig. 4. Continuous controlled valve ([9])

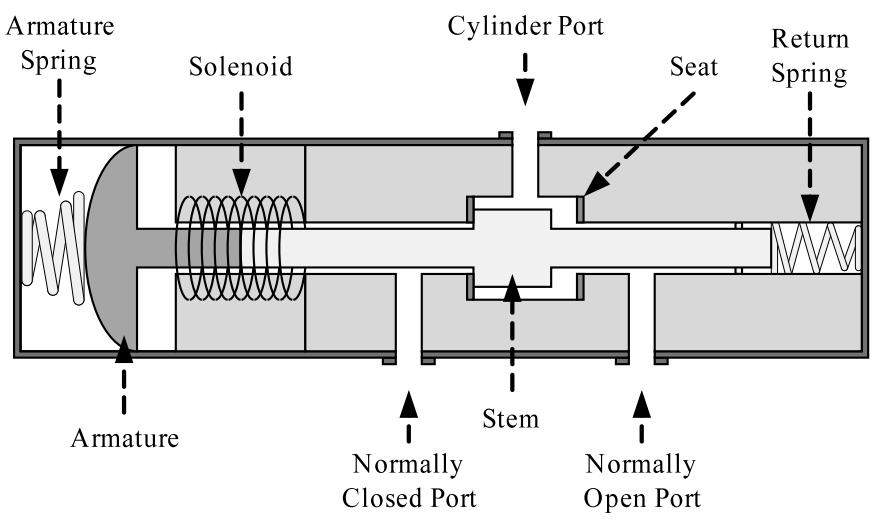

Fig. 5. Three-way two-position solenoid valve.

output (3 to $15 \mathrm{psig}$ ). The IPT is essentially a pressure regulator that is controlled by a current signal. Supply pressure is connected to the input port, and, the regulated output pressure will change depending on the current signal.

Controllable gas leaks are introduced through a set of proportional valves. These valves combine a solenoid valve with an electronics package that digitally modulates the control signal to provide analog proportional control. These are twoway normally-closed valves and operate on $24 \mathrm{~V} \mathrm{dc}$, powered through the power supply or the batteries.

The data from the different sensors is collected using an 8slot NI cDAQ-9188 Gigabit Ethernet chassis which is designed for remote or distributed sensor and electrical measurements. In the experimental testbed for safety requirements all the controls and data acquisition activities are done remotely hence the specific chassis is selected for the testbed. A single NI CompactDAQ chassis can measure up to 256 channels of sensor signals, analog I/O, digital I/O, and counter/timers with an Ethernet interface back to a host machine. The details of the cards used are as follows:

1) NI 9211 4-Channel, $14 \mathrm{~S} / \mathrm{s}, 24-\mathrm{Bit}, 80 \mathrm{mV}$ thermocouple input module to sense the temperature of the batteries
2) NI 920532 single-ended or 16 differential analog inputs 16-bit resolution; $250 \mathrm{kS} / \mathrm{s}$ aggregate sampling rate $200 \mathrm{mV}, 1,5$, and $10 \mathrm{~V}$ programmable input ranges for pressure sensing

3) NI $94018 \mathrm{Ch}, 5 \mathrm{~V} / \mathrm{TTL}$ high-speed bidirectional digital I/O module. 8-channel, $100 \mathrm{~ns}$ ultrahigh-speed digital I/O $5 \mathrm{~V} / \mathrm{TTL}$, sinking/sourcing digital I/O for digital control

4) NI 926416 channel analog output module. $25 \mathrm{kS} / \mathrm{s} / \mathrm{ch}$ simultaneous output hot-swappable operation 60 VDC isolation with D-SUB version card for controlling the proportional control valves.

All the operations for the cDAQ-9188 are controlled through a LabVIEW-designed interface from where the user can have access to all the input data and output control.

\section{Pneumatic Gas Leak Faults}

As discussed earlier, the prognostic demonstration testbed is an apparatus used to showcase remaining life prediction in the context of cryogenic refueling operations. The most common type of fault is a leak of pneumatic gas. To demonstrate valve prognostics for leaks we select several locations throughout the pressure lines for which leaks may appear in order to emulate faults. The injection is accomplished by adding a bypass line with a proportional solenoid valve in the pneumatics lines as illustrated in Figure 2. The bypass valves are remotely operated and allow control over how much they can be opened in order to control the leakage rate and support desired damage progression profiles. Table I summarizes the different faults that may appear in the system and their effect on the components. The following subsections describe how these fault modes can be emulated on the testbed.

\section{A. Solenoid Valve and DV Leak Faults}

Figure 6 illustrates the setup for faults that can be injected in the solenoid valve when energized, to study the degradation effects on the operation of the DV. As illustrated, the leakage faults can be injected at the NO and NC seat ports using the bypass valve V1 which affects the DV operation due to decreased supply pressure. The leak through V1 emulates a leak at the cylinder port or, when energized, a leak across the NO seat (see Table I).

Similarly, Figure 7 illustrates the setup for faults that can be injected in the solenoid valve when de-energized, to study the degradation effects on the operation of the DV. A leakage fault can be injected at the NO and NC seat ports using the bypass valve V2 which affects the DV operation due to decreased supply pressure. In both the injected faults, the amount the valve will open depends on steady-state pressure and whether it produces enough force to overcome the spring force. The steady-state value and how long it takes to get there depends on orifice sizes and the leak rate. The amount the valve will close depends on steady-state pressure and if this decreases the gas force enough for the spring force to overcome it.

In addition to the leakage faults, a fault injected in the coils will change the operational timing of the seat opening. 
TABLE I

FAult InJeCtion Matrix For PROgnostics Testbed

\begin{tabular}{|c|c|c|}
\hline Component & Fault Mode & Effects \\
\hline \multirow[t]{4}{*}{ Solenoid Vale } & Leak across NC seat & $\begin{array}{l}\text { If SV energized, and DV valve is open, no effect; if DV valve is closed, no effect. If SV } \\
\text { de-energized, and DV valve is closed, DV valve potentially opens; if DV valve is open, } \\
\text { DV closes more slowly. }\end{array}$ \\
\hline & Leak across NO seat & $\begin{array}{l}\text { If SV energized, and DV valve is open, loses pressure and DV can start to close; if DV } \\
\text { valve is closed, it will open more slowly. If SV deenergized, and DV valve is closed, no } \\
\text { effect; if DV valve is open, will close more slowly. }\end{array}$ \\
\hline & Leak at cylinder port & Same as leak across NC seat. \\
\hline & Coil resistance degradation & Solenoid current will increase (transient and steady-state). SV timing will change. \\
\hline DV Valve & $\begin{array}{l}\text { Pneumatic gas leak at valve port } \\
\text { Spring wear }\end{array}$ & $\begin{array}{l}\text { Same effects as leak at SV cylinder port or leak across NO seat. } \\
\text { Valve opens faster, closes slower. If spring gets weak enough, valve will not close } \\
\text { completely. }\end{array}$ \\
\hline I/P Transducer & $\begin{array}{l}\text { Leak at input port } \\
\text { Leak at output port } \\
\text { Coil resistance increase } \\
\text { Spring wear }\end{array}$ & $\begin{array}{l}\text { Lowers regulated pressure } \\
\text { Lowers regulated pressure } \\
\text { Lowers regulated pressure } \\
\text { Increase or decrease regulated pressure depending on which spring - return spring } \\
\text { decrease would increase output pressure }\end{array}$ \\
\hline CV Valve & $\begin{array}{l}\text { Pneumatic gas leak at supply pressure port } \\
\text { Pneumatic gas leak at signal pressure port }\end{array}$ & $\begin{array}{l}\text { Lower supply pressure so valve may not open fully, open more slowly } \\
\text { Lowers regulated pressure }\end{array}$ \\
\hline
\end{tabular}

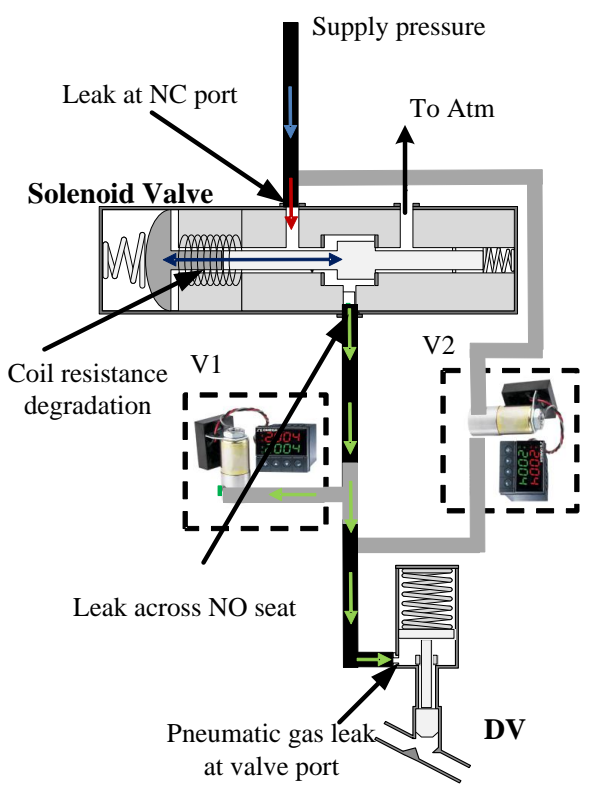

Fig. 6. Solenoid valve leak fault injection when energized on DV valve

Injecting a increase in resistance will increase the solenoid current (transient and steady-state) and hence affect the SV timing and hence the DV timing.

\section{B. IPT and CV Valve Leak Faults}

Figure 8 illustrates the setup for faults that can be injected through the IPT and bypass valves, to study the degradation effects on the operation of the CV. As can be seen, a leakage fault can be injected at the supply pressure port through bypass

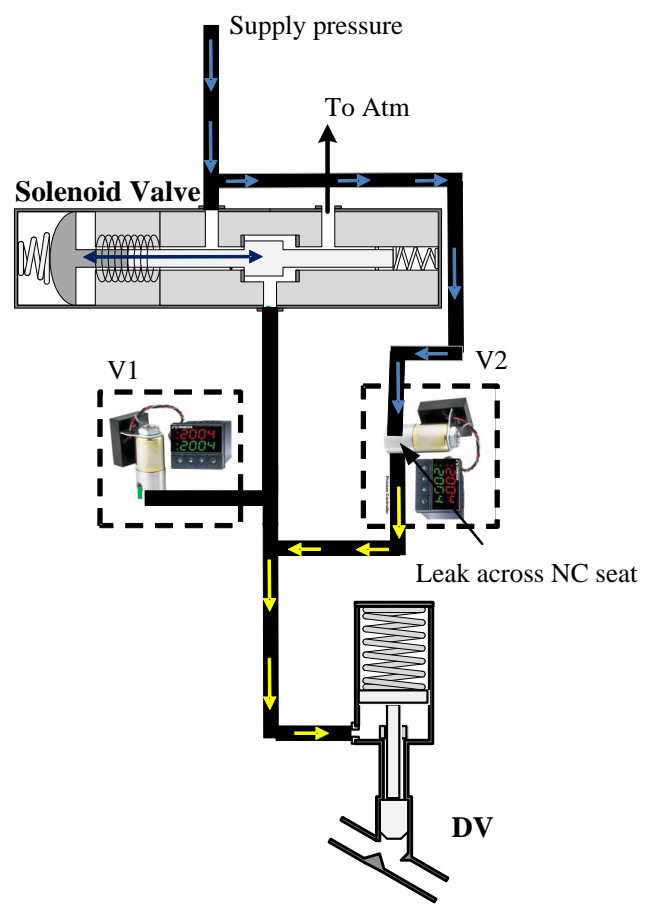

Fig. 7. Solenoid valve leak fault injection when de-energized on DV valve

valve V4. Any changes in the supply pressure will have an effect on the opening and closing of the $\mathrm{CV}$ valve. A leak in the signal pressure line from the IPT to the $\mathrm{CV}$ can also be injected through V3. Since the IPT modulates the amount of pressure at its output depending upon the control signal, a leak on the signal line will reduce the signal pressure going to the 


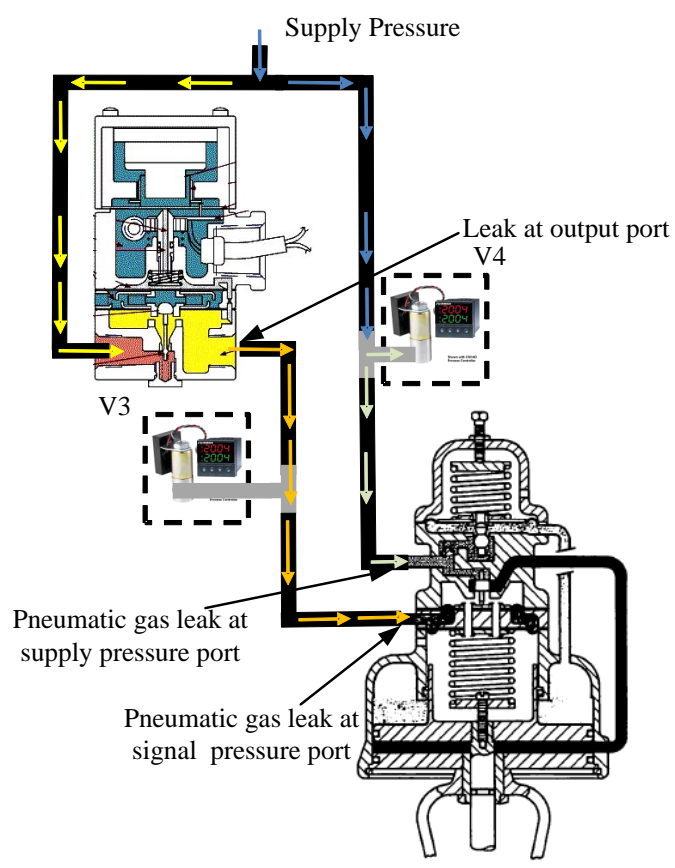

Fig. 8. CV pneumatic leak faults

$\mathrm{CV}$ and modify its position.

\section{BATTERIES}

As part of a backup power supply source, Li-ion batteries are used for powering the solenoid valve and IPT. Theoretically the cell has a voltage of around $4.2 \mathrm{~V}$ when fully charged. The terminal voltage of the battery rises/falls with a charge/discharge cycle, respectively. So to get a total dc voltage of around $24 \mathrm{~V}$ to operate the valves we connect 6 batteries in series. The batteries can provide a backup for a limited amount of time and as the charge in the battery starts decreasing the output power decreases. In addition to this, over the period of time the battery degrades and its ability to hold charge decreases. This degradation in performance of the battery will affect the operation of the powered sub-systems, which eventually will have a cascading effect on the control valves. To avoid any incomplete operation of critical operations or accidents requires implementation of an advanced health monitoring and predictive capabilities methodology. To implement such technologies, it is crucial to understand how batteries work and to capture that knowledge in the form of models that can be used by monitoring, diagnosis, and prognosis algorithms.

In order to predict end-of-discharge, we have developed an electrochemistry-based model of Li-ion batteries [10] that (i) captures the significant electrochemical processes, (ii) is computationally efficient, (iii) captures the effects of aging, and (iv) is of suitable accuracy for reliable EOD prediction in a variety of usage profiles. The model can be considered an electrochemical engineering model, but unlike most such models found in the literature, we make certain approximations that allow us to retain computational efficiency.

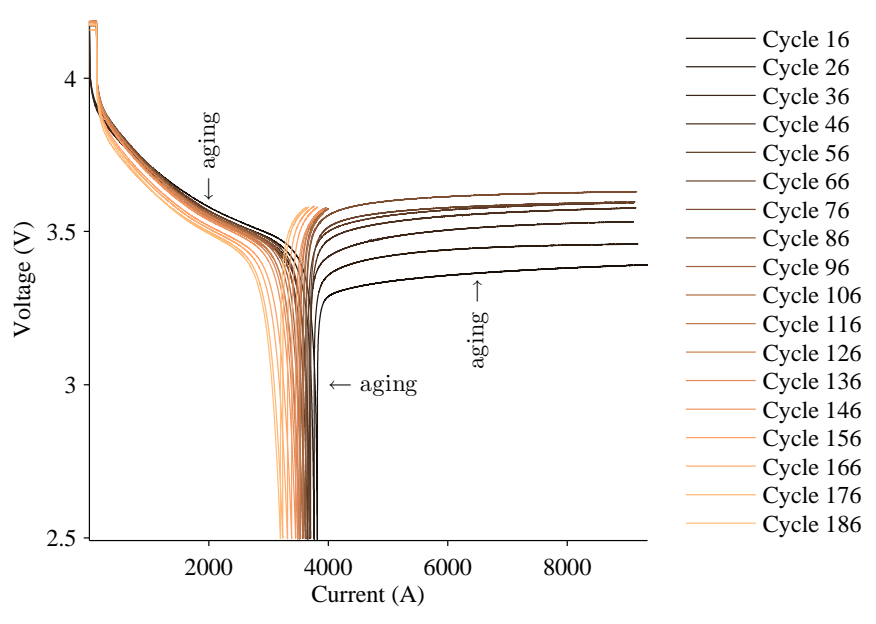

Fig. 9. Measured 2 A discharge curves with battery aging.

The end of life of a battery depends on the chemistry, discharge-charge cycling, temperature, and storage conditions, among other factors. Atmospheric temperature affects the operating performance of the batteries. At low temperatures, ionic diffusion and migration could be hindered and also damaging side reactions like lithium plating may take place. High temperatures cause corrosion and generation of gases leading to an increase in internal pressure. As the battery ages, degradation results in the decrease of the observed battery capacity. This is primarily due to a loss of mobile ions due to parasitic or side reactions and an increase in the internal resistance. Internal resistance leads to ohmic losses that generate heat and accelerate the aging process. Some relevant physical aging mechanisms in the electrodes are solidelectrolyte interface (SEI) layer growth, lithium corrosion, formation of plating on lithium, and contact loss due to the formation of the SEI layer.

To study the charge/discharge dynamics of batteries we characterized the batteries by taking voltage measurements under constant current charge/discharge conditions. The rate of charge/discharge is measured relative to the battery capacity C. For example, a $0.1 \mathrm{C}$ discharge rate for a $5 \mathrm{Ah}$ battery is 0.5 A. Fig. 9 shows 2 A discharge curves with the battery at different ages. The EOD point moves earlier in time due to diminished capacity. The voltage drops down during discharge due to increased resistance. Steady-state voltage after discharge increases because the battery is not discharged as fully due to the increased resistance and the battery hitting EOD before then.

The detailed implementation of the derived electrochemistry model and algorithms for li-ion batteries are discussed in [10].

\section{CONCLUSION}

In this paper, we presented a prognostics demonstration testbed for cryogenics propellant loading. Using the sensor data from the injected faults we plan to implement prognostics algorithms such that we are able to make EOL predictions that 
are accurate. The test bed will help in studying realistic degradation and failure effects on the propellant loading systems and implementing prognostic methodologies to such systems will enable operations crews to take effective operations and maintenance-related decisions, thus efficiently streamlining operational and mission-level activities.

The current testbed is limited in several ways that provides the basis for future work. For one, we are currently only looking at prominent faults that could be injected in the system. Other more complicated faults are not included in this study. As we better understand the system and the ability to model system better we plan to incorporate those faults. Further integrating the testbed with the actual cryogenic propellant system will be an interesting step to study the effects of injected faults on the complete system and then implementing health monitoring and prognostics methodologies for making accurate end-of-life predictions. Earlier, much work has been done related to cryogenic loading systems but it was not carried out with prognostics requirements and applications in mind. The current work is an initial step towards implementing these methodologies to industrial process plants and similar equipments.

\section{ACKNOWLEDGMENT}

This work was funded by the NASA Automated Cryogenic Loading Operations (ACLO) project under the Office of the Chief Technologist (OCT) of Advanced Exploration Systems (AES), and by the Advanced Ground Systems Maintenance
(AGSM) Project under the Ground Systems Development and Operations program.

\section{REFERENCES}

[1] N. P. Zeitlin, G. R. Clements, S. J. Schaefer, M. K. Fawcett, and B. L. Brown, "Ground and launch systems processing technologies to reduce overall mission life cycle cost," in 2013 IEEE Aerospace Conference, Mar. 2013.

[2] C. Goodrich, S. Narasimhan, M. Daigle, W. Hatfield, R. Johnson, and B. Brown, "Applying model-based diagnosis to a rapid propellant loading system," in Proceedings of the 20th International Workshop on Principles of Diagnosis, Jun. 2009, pp. 147-154.

[3] M. Daigle and K. Goebel, "A model-based prognostics approach applied to pneumatic valves," International Journal of Prognostics and Health Management, vol. 2, no. 2, Aug. 2011.

[4] — "Model-based prognostics with concurrent damage progression processes," IEEE Transactions on Systems, Man, and Cybernetics: Systems, vol. 43, no. 4, pp. 535-546, May 2013.

[5] B. Saha and K. Goebel, "Modeling Li-ion battery capacity depletion in a particle filtering framework," in Proceedings of the Annual Conference of the Prognostics and Health Management Society 2009, Sep. 2009.

[6] M. Daigle, B. Saha, and K. Goebel, "A comparison of filter-based approaches for model-based prognostics," in Proceedings of the 2012 IEEE Aerospace Conference, Mar. 2012.

[7] M. Daigle, A. Saxena, and K. Goebel, "An efficient deterministic approach to model-based prediction uncertainty estimation," in Annual Conference of the Prognostics and Health Management Society, Sep. 2012, pp. 326-335.

[8] M. Daigle, A. Bregon, and I. Roychoudhury, "A distributed approach to system-level prognostics," in Annual Conference of the Prognostics and Health Management Society, Sep. 2012, pp. 71-82.

[9] "Er4000 masoneilan domotor actuator instructions," 1991, rev. B8/84.

[10] M. Daigle and C. Kulkarni, "Battery prognostics using electrochemistrybased models," October 2013, manuscript submitted for publication. 